\title{
A comparative study of Monte Carlo-coupled depletion codes applied to a Sodium Fast Reactor design loaded with minor actinides
}

\author{
R. Ochoa ，M. Vázquez ，F. Álvarez-Velarde ，F. Martín-Fuertes ，N. García-Herranz ，D. Cuervo'
}

\begin{abstract}
A B S T R A C T
Minor actinides (MAs) transmutation is a main design objective of advanced nuclear systems such as generation IV Sodium Fast Reactors (SFRs). In advanced fuel cycles, MA contents in final high level waste packages are main contributors to short term heat production as well as to long-term radiotoxicity. Therefore, MA transmutation would have an impact on repository designs and would reduce the environment burden of nuclear energy. In order to predict such consequences Monte Carlo (MC) transport codes are used in reactor design tasks and they are important complements and references for routinely used deterministic computational tools. In this paper two promising Monte Carlo transport-coupled depletion codes, EVOLCODE and SERPENT, are used to examine the impact of MA burning strategies in a SFR core, 3600 MWth. The core concept proposal for MA loading in two configurations is the result of an optimization effort upon a preliminary reference design to reduce the reactivity insertion as a consequence of sodium voiding, one of the main concerns of this technology. The objective of this paper is double. Firstly, efficiencies of the two core configurations for MA transmutation are addressed and evaluated in terms of actinides mass changes and reactivity coefficients. Results are compared with those without MA loading. Secondly, a comparison of the two codes is provided. The discrepancies in the results are quantified and discussed.
\end{abstract}

\section{Introduction}

In addition to the traditional roles in a medium or large Sodiumcooled Fast Reactors (SFRs) for electricity production and plutonium breeding, a new tentative strategy is nowadays pursued concerning minor actinide (MA) transmutation. This strategy would allow minimization of already accumulated nuclear wastes coming from LWR open fuel cycles, which in turn may have a positive impact on final repository requirements. However, it is well known that MA loading in a reactor has a large impact on aspects such as safety parameters (i.e. Doppler coefficient, $\beta_{\text {eff, void effect), helium }}$ production in the fuel, mechanical fuel behaviour under irradiation, and batch cycle considerations that need a careful assessment.

Different alternatives for MA loading have been studied in the past (see Section 2.2). The performed studies show that the introduction of MA in the reactor fuel deteriorates the core reactivity coefficients, where the magnitude of impact depends on the MA content and the type of recycle approach - standard homogeneous or heterogeneous management or intermediate solutions. But, as pointed among others in (Naganuma et al., 2010), (Palmiotti et al., 2011), there is no fixed limit of MA loading, but for each particular system configuration a limit should be defined according to different aspects, such as safety limits for reactivity coefficients. Therefore, optimized core designs around basic designs could limit the potential negative impact of the MA content in the reactivity effects, and then the limits should be carefully assessed for each particular configuration.

This work has been done in the frame of the CP-ESFR Project (Fiorini et al., 2011). One of the objectives in the project was to explore different designs and operation strategies of Sodium-cooled Fast Reactors in order to enhance the nominal core performances as well as the core safety mostly in the frame of unprotected transients. Optimization studies were carried out in the project, and an optimized oxide core with a reduced sodium void reactivity was proposed (see Section 2), for which an assessment of the MA transmutation possibilities is of major interest.

From the computational point of view, most of the performed studies in CP-ESFR and other projects used deterministic calculation approaches like ERANOS (Rimpault et al., 2002) to assess the effects of MA recycling on the core performances (Buiron et al., 2009). Deterministic methods for transport calculations utilize spatial, angular and energy discretization, as well as the prior development of appropriate multi-group constants. Nevertheless, due to the acceptable computational time, they are the most common approach nowadays for fast reactors neutron analysis. 
However, the increased relevance of Monte Carlo transport codes, mainly due to the improvement of computing performances, makes that such codes are starting to be an alternative for design calculations (Yang, 2012). Its integration with depletion modules provides tools able to model very detailed and complex threedimensional core geometries using continuous-energy cross-section data. This approach eliminates the concern of generation of multigroup cross sections, which remains one of the fundamental problems in fast reactor physics because of the need for accurate self-shielding treatment (Yang, 2012).

Since the 90s, different depletion computational systems have been developed around the world coupling a Monte Carlo neutron transport code with a depletion code. This is the case among others of MCNPX 2.6.0 (Fensin et al., 2010), MONTEBURNS (Trellue and Poston, 1999), MOCUP (Moore et al., 1995) and MCODE (Xu and Hejzlar, 2008). For the purpose of this work, two codes were selected: (i) EVOLCODE (Âlvarez-Velarde et al., 2007), which automatically links the MCNPX Monte Carlo transport code with the depletion code ORIGEN; (ii) SERPENT MC transport code (Leppänen, 2011), which incorporated the depletion capability by the coupling with a burnup algorithm. EVOLCODE is being developed at CIEMAT since some years ago, and related activities include a number of successful validation exercises (Álvarez-Velarde et al., 2011), (Álvarez-Velarde et al., 2012). SERPENT is recently being used at UPM and it is expected to obtain very similar results as MCNPX for criticality calculations, with faster execution. The code is released with an open source.

This paper describes in Section 2 the core design including the proposed MA loading patterns, and in Section 3 the computational tools. The comparison between EVOLCODE and SERPENT codes is covered in Section 4, providing additional information regarding MA impact on core performances. Finally, Section 5 summarizes the main conclusions.

\section{Reference core configuration}

\subsection{Basis optimized core description}

The selected core for the analysis in the CP-ESFR European Project is the so called CONF2 core (Rineiski et al., 2011). It is a 3600 MWth Sodium-cooled Fast Reactor that consists of two separated driver fuel regions of 225 inner fuel assemblies (FAs) and 228 outer fuel assemblies with 271 fuel pins/FA. The assembly pitch is $21.08 \mathrm{~cm}$ while the fuel pellet diameter is $0.943 \mathrm{~cm}$. The FAs are loaded with MOX fuel with a small fraction of ${ }^{241} \mathrm{Am}$ coming from ${ }^{241} \mathrm{Pu}$ decay during fresh fuel storage. The inner and outer regions present different $\mathrm{Pu}$ contents $(14.76 \mathrm{wt} \%$ and $17.15 \mathrm{wt} \%$ respectively) in order to provide low peaks of the neutron flux and local power. The considered isotopic vectors, taken from (Buiron et al., 2009), are as follows:

- Uranium isotopic composition: ${ }^{235} \mathrm{U} /{ }^{238} \mathrm{U}=0.25 / 99.75$ (wt \%).

- Plutonium isotopic composition: ${ }^{238} \mathrm{Pu} /{ }^{239} \mathrm{Pu} /{ }^{240} \mathrm{Pu} /{ }^{241}$ $\mathrm{Pu} /{ }^{242} \mathrm{Pu} /{ }^{241} \mathrm{Am}=3.57 / 47.39 / 29.66 / 8.23 / 10.37 / 0.78$ (wt\%).

Three radial rings of reflector assemblies surround the active core. The axial layout of the optimized core presents, just above the active core, a large sodium plenum. Further above in the axial direction there is a layer of neutron absorbent material (boron carbide) and another layer of steel reflector. In the lower part, just below the active core, there is a fertile region in order to provide neutron absorptions.

The control rod system is composed of 9 DSD (Diverse Shutdown Device, $90 \% \mathrm{w}{ }^{10} \mathrm{~B}$ ), located in the second ring of control assemblies, and 24 CSD (Control and Shutdown Device, $\sim 19.9 \% \mathrm{w}$

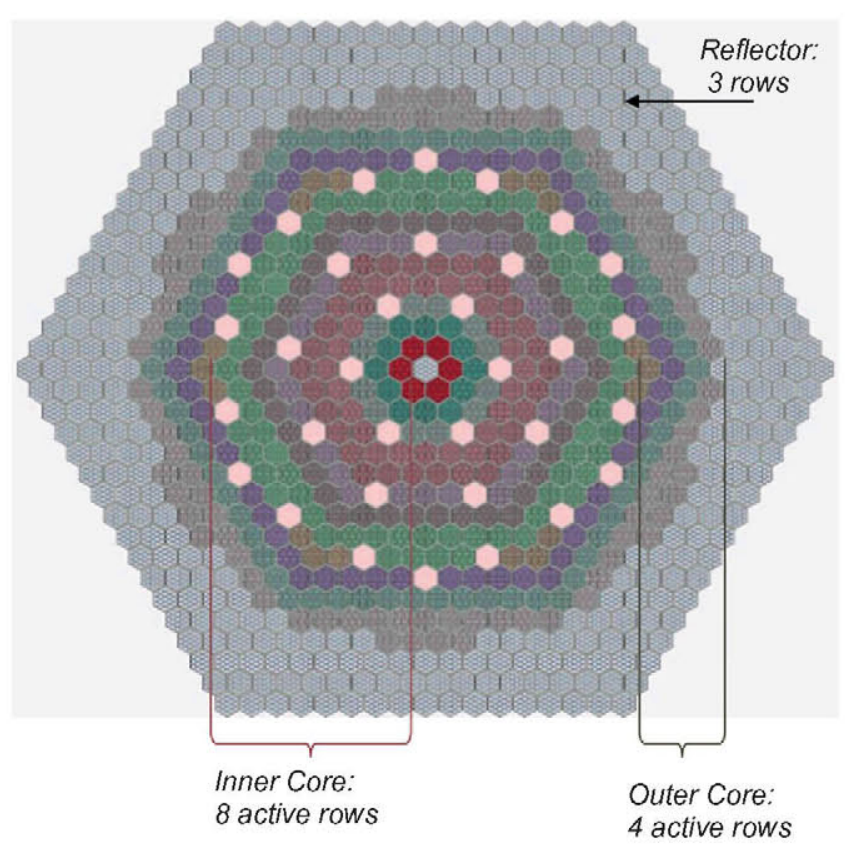

Fig. 1. Cross section view of the optimized ESFR core, SERPENT post-processing (a very similar figure is obtained with MCNPX post-processing).

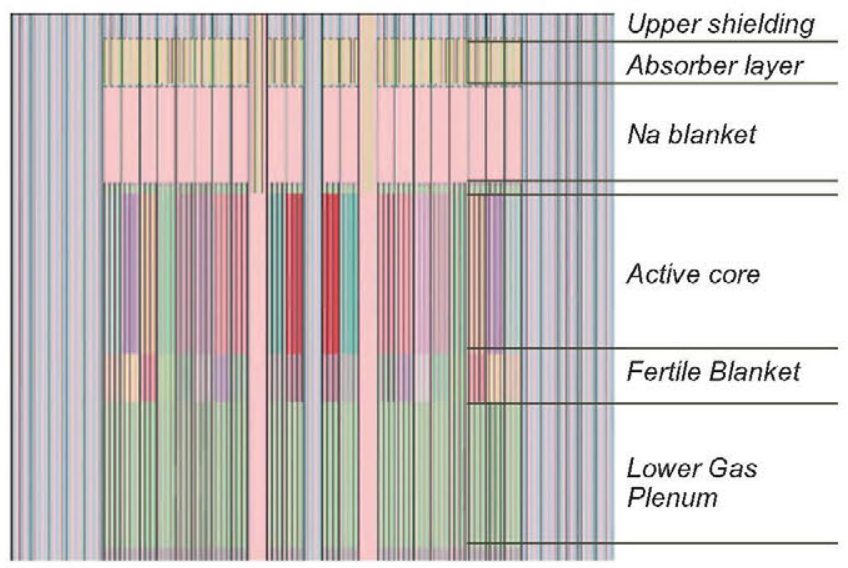

Fig. 2. Vertical view of the optimized ESFR core, SERPENT post-processing.

$\left.{ }^{10} \mathrm{~B}\right)$. The control assemblies were considered withdrawn for the purpose of the analysis. Cross section and vertical views of the reactor core are shown in Figs. 1 and 2 respectively. In the vertical view, the following parts can be distinguished from the top to the bottom: the upper shielding structure, absorber layer, sodium plenum, upper plug, upper gas plenum, active core, fertile blanket, lower gas plenum and lower plug.

\subsection{MA loading patterns}

A number of core configurations with MA loading has been proposed and assessed by researchers around the world in the last years. A short general classification is the following:

- Homogeneous mixture of MA distributed throughout the whole core fuel, i.e., a mixture of fissile (Pu), fertile (U) and MA. The Pu content is tuned, per reactor zones, according to reactivity swing, breeding ratio and power peak requirements, while the 
MA content is typically low, below $4 \%$ or $5 \%$ weight of Heavy Metal (HM) (Ohki et al., 2007), (Zhang et al., 2010). There are also studies with MA contents higher than $5 \%$ in the fresh fuel (Palmiotti et al., 2011), (Rineiski et al., 2011).

- Heterogeneous recycling approach, where MA loading is concentrated in radial fuel assemblies at high contents, 10 $30 \mathrm{wt} \%$, mixed with $U$ fertile material, and no Pu content; the $\mathrm{Pu}$ is only loaded in the core driver in this configuration (Buiron et al., 2007). A variant of this case is to dedicate a lower or upper axial blanket to MA. In that configuration the purpose is to take benefit from the neutrons leaking from the reactor for MA transmutation and $\mathrm{Pu}$ breeding. As the neutronic importance is low in the reactor periphery, the impact on neutronic parameters due to MA is small.

- Intermediate radial or axial blankets with high MA content, similar to the previous fully heterogeneous configuration, but where some $\mathrm{Pu}$ content is loaded also in the blanket together with the uranium and MA (Buiron et al., 2009). The purpose is to avoid large decrease of the peaking factors in those positions close to the ones developing power. This kind of solutions can be considered as homogeneous since MA are mixed with the driver fuel, and heterogeneous since only some limited core regions are involved.

- Special fuel subassembly targets with high contents of MA and no fertile material. The MA mixture is contained in a neutronically inert matrix, metallic or ceramic material. These targets are distributed throughout the reactor in a certain number of fuel assembly positions in the core. On the other hand, some rods of the target fuel assembly may be occupied by moderating rods (e.g., $\mathrm{ZrH}_{\mathrm{x}}$ ), as transmutation rate can increase when the local spectrum is softened (Bays et al., 2011).

Behind such configurations there are several possibilities of spent fuel reprocessing and separation. They are in general advanced processes at laboratory stage which could reach technological readiness levels after some decades (OECD, 2004), (OECD, 2010). For instance, all transuranics can be separated together in a single stream, or all MA can be extracted together in a stream while $\mathrm{Pu}$ is separated in a different one, or $\mathrm{Pu}$ and $\mathrm{Np}$ are separated together or $\mathrm{Cm}$ is extracted from the MA stream and put aside for specific management as a consequence of originating high irradiation levels (OECD, 2012).

Among all above possibilities, the first two recycling models are explored in this paper for the CP-ESFR optimized CONF2 core:

- The homogenous distribution of MA with other fuel components, with 4 wt\% of MA content, is HOM4 case. The lower axial blanket presented in Section 2.1 is assumed also $4 \mathrm{wt} \% \mathrm{MA}$ loaded (and no Pu content). This case is intended to provide insights in line with moderate transmutation values in homogeneous loading compatible with affordable deterioration of safety parameters.

- The heterogeneous core model, with a radial blanket of MA (located surrounding the last row of outer fuel, in the place of the first reflector row) and depleted uranium, with $20 \mathrm{wt} \%$ of MA enrichment is HET2 case. Higher contents of MA can be considered too much optimistic as the curium content complicates fuel management.

In both cases oxide fuel form is assumed in driver and blanket regions. A typical MA vector coming from the recycling of LWR spent fuel in the transitional stage from light water reactors to fast reactors is as follows, and will be the one considered for this analysis: ${ }^{237} \mathrm{~Np} /{ }^{241} \mathrm{Am} /{ }^{242 \mathrm{~m}} \mathrm{Am} /{ }^{243} \mathrm{Am} /{ }^{242} \mathrm{Cm} /{ }^{243} \mathrm{Cm} /{ }^{244} \mathrm{Cm} /{ }^{245} \mathrm{Cm} /$ ${ }^{246} \mathrm{Cm}=16.86 / 60.62 / 0.24 / 15.7 / 0.02 / 0.07 / 5.14 / 1.26 / 0.09$ (wt\%).

\section{Computational tools and modelling}

\subsection{EVOLCODE}

EVOLCODE 2.0 (Álvarez-Velarde et al., 2007) is a CIEMAT development to automatically couple MCNPX (Pelowitz, ed., 2008) and ORIGEN (RSICC, 1999) or ACAB codes (Sanz et al., 2008), both of them based on the matrix exponential method. Fuel material homogeneously evolves along several burn-up steps for cells that can be selected by the user. The first stage solver is the MCNPX code, which allows an important degree of the heterogeneity description in the reactor core model. In a second computational stage, EVOLCODE obtains a single-group cross section for materials in every core cell under MCNPX predicted spectra conditions and feeds ORIGEN code (alternatively $\mathrm{ACAB}$ ) to provide the inventory evolution under irradiation for a user-input burnup step. After this stage, EVOLCODE2 automatically generates a second MCNPX input with updated material cards to estimate new spectra and relative fluxes for the next burn-up step.

EVOLCODE uses a predictor-corrector method based on constant neutron flux value along the burnup step. A first flux value is tentatively assumed to obtain a preliminary estimation of the irradiation results. This value is based on the normalization of MCNPX results (accounting for all reactor cells) before irradiation. A provisional irradiation is performed and the new composition generally leads to end of step power values relatively far from specified. Therefore, the flux is corrected to match the required power for the definitive step. The assumed macroscopic cross sections to start irradiation are always taken at the beginning of step (Álvarez-Velarde, 2011).

A specific issue is the branching ratio in metastable isotopes, for which EVOLCODE considers energy-dependent branching ratios taken from the JEFF 3.1.1 data library.

CIEMAT activities concerning EVOLCODE 2.0 are completed with systematic comparison of predictions to real irradiations results (Álvarez-Velarde et al., 2012), as well as cross-checking to other codes.

In this paper the $\beta_{\mathrm{eff}}$ is calculated using a modified MCNPX version developed at CIEMAT. It is based on a generalization of the Meulekamp method (Meulekamp and Van der Marck, 2006): when a fission takes place it is further checked if a delayed neutron produced that fission (all neutrons were labeled prompt or delayed at birth). Then, the total number of fissions originated by delayed neutrons is divided by total fissions generated by all neutrons, which directly leads to the $\beta_{\text {eff }}$ estimation. Among other internal exercises, the modified MCNPX version to compute $\beta_{\text {eff }}$ has been crosschecked to other codes in the frame of the CP-ESFR Project with good agreements.

\subsection{SERPENT}

SERPENT (Leppänen, 2011) is a three-dimensional continuousenergy Monte Carlo reactor physics burnup calculation code developed at the VTT Technical Research Centre of Finland since 2004. The code is specialized in lattice physics calculations. The neutron transport is based on a combination of conventional surface-tosurface ray-tracing and the Woodcock delta-tracking method. Burnup depletion equations are solved using the matrix exponential method CRAM (Pusa and Leppänen, 2010 and Pusa, 2011), providing a robust and accurate solution with a very short computation time. A comparison between CRAM, ORIGEN solver and other TTA (Truncated Taylor Approximation) methods proved the advantages of the CRAM method in terms of accuracy and running time, thanks to the mathematical approach behind CRAM (Isotalo and Aarnio, 2011a). 
SERPENT also uses a predictor-corrector method. In this case, after a provisional irradiation step (predictor step), a neutron transport problem is solved at end of step (EOS), and the average fluxes and cross sections between the BOS (beginning of step) and EOS are employed to recalculate the EOS compositions (Isotalo and Aarnio, 2011b). Regarding branching ratios, SERPENT takes by default constant values.

SERPENT computes directly the effective delayed neutron parameters using the same Monte Carlo method developed at the NRG (Meulekamp and Van der Marck, 2006). It is reported that compared to other coupled Monte Carlo burnup calculation codes based on MCNP like MONTEBURNS, the calculation time required by SERPENT to achieve a given statistical uncertainty has been reduced by a factor of $5-15$, by evenly subdividing the cross section energy grid (Zhang et al., 2010).

A burn-up calculation using SERPENT code requires a lot of memory storage capacity, which is one of the main drawbacks of the code. As a consequence, new improvements have been implemented in the new version of the code, SERPENT2 (Leppänen and Isotalo, 2012), currently in a beta-testing phase.

\subsection{Modelling}

Three-dimensional MCNPX and SERPENT geometry models were developed (CONF2, CONF2-HOM4, CONF2-HET2), from the

Table 1

Computational characteristics.

\begin{tabular}{rll}
\hline & $\begin{array}{l}\text { SERPENT (using } \\
\text { one core) }\end{array}$ & $\begin{array}{l}\text { EVOLCODE (parallel } \\
128 \text { cores) }\end{array}$ \\
\hline $\begin{aligned} \text { Calculation time of a } \\
\text { single burnup step }\end{aligned}$ & $\sim 8 \mathrm{~h}$ proc $3 \mathrm{GHz}$ & $\sim 2 \mathrm{~h} 15 \mathrm{~m}$ proc $3 \mathrm{GHz}$ \\
Total calculation time & $24 \mathrm{~GB} \mathrm{RAM}$ & $(2 \mathrm{~GB} /$ core) \\
\hline
\end{tabular}

lower plug up to the upper shielding layer in the reactor, neglecting the head and foot of the sub-assemblies. Helical wire wrap spacers between fuel pins were considered as merged in the cladding.

A single batch approach for the whole irradiation period is assumed. In this approach, a cycle length of 2050 equivalent full power days (EFPDs) is computed starting from the fresh core. The 2050 EFPD cycle is divided into five periods of 410 EFPD. Though no batch reloading is assumed, real core reloading would be envisaged each 410 EFPD. As a first approach, 820 and 1230 EFPD can be used as a rough estimate of core conditions for beginning and end of equilibrium cycles (BOECs, EOECs), as the cumulated fission product population is representative. Around 1100 independent isotopes are tracked with EVOLCODE and around 500 by SERPENT for the particular models under study (the isotopes not considered in this set are assumed reasonably neglected in terms of masses and macroscopic cross section values).

A total number of 150,000 neutron histories and 200 active cycles were employed in both codes. The average statistical uncertainties obtained in $k_{\text {eff }}$ are 10 and $8 \mathrm{pcm}$ respectively for SERPENT and EVOLCODE. In both codes JEFF3.1.1 cross section library was used for neutron reactions under the same assumed material working temperatures. Fission yields were also taken from JEFF-3.1.1 library.

For isotope depletion, the burnable region was divided in a number of computational cells, intersection of 12 radial rings (eight for the inner core, four for the outer core) and different axial levels depending on the model:

- In the analysis with SERPENT, only two axial levels were considered, one for the active core, $100 \mathrm{~cm}$ high, and one axial level for the lower blanket, $30 \mathrm{~cm}$ high. This is a consequence of the high memory requirements for a burn-up calculation using SERPENT, which makes a finer refinement not possible in the machine employed (Table 1).

Table 2

Comparison of transmutation performances.

\begin{tabular}{|c|c|c|c|c|c|c|}
\hline & \multicolumn{2}{|l|}{ CONF2 } & \multicolumn{2}{|l|}{ HOM4 } & \multicolumn{2}{|l|}{ HET2 } \\
\hline & SERPENT & EVOLCODE & SERPENT & EVOLCODE & SERPENT & EVOLCODE \\
\hline \multicolumn{7}{|c|}{ Charged mass $(\mathrm{kg})$} \\
\hline $\mathrm{U}$ & 87190.4 & 87158.7 & 80676.1 & 80659.7 & 101514.2 & 101483.7 \\
\hline $\mathrm{Np}$ & 0.0 & 0.0 & 650.1 & 649.9 & 603.9 & 603.7 \\
\hline $\mathrm{Pu}$ & 11855.9 & 11849.7 & 11856.8 & 11854.4 & 11855.9 & 11849.7 \\
\hline $\mathrm{Am}$ & 93.2 & 93.2 & 2951.8 & 2951.0 & 2835.5 & 2834.7 \\
\hline $\mathrm{Cm}$ & 0.0 & 0.0 & 253.8 & 253.6 & 235.7 & 235.6 \\
\hline MA & 93.2 & 93.2 & 3855.7 & 3854.5 & 3675.1 & 3673.9 \\
\hline \multicolumn{7}{|c|}{ Discharged mass $(\mathrm{kg})$} \\
\hline $\mathrm{U}$ & 78017.4 & 77948.7 & 72632.4 & 72605.1 & 92160.2 & 92089.6 \\
\hline $\mathrm{Np}$ & 44.1 & 43.3 & 406.0 & 411.1 & 583.8 & 583.5 \\
\hline $\mathrm{Pu}$ & 13120.8 & 13209.1 & 13417.4 & 13466.4 & 13711.6 & 13799.3 \\
\hline $\mathrm{Am}$ & 338.8 & 338.3 & 1900.4 & 1924.7 & 2701.7 & 2703.4 \\
\hline $\mathrm{Cm}$ & 66.1 & 65.6 & 472.0 & 467.5 & 333.6 & 332.0 \\
\hline MA & 449.0 & 447.2 & 2778.4 & 2803.4 & 3619.1 & 3618.9 \\
\hline \multicolumn{7}{|c|}{ Transmutation rate $(\%)$ at $E O L$} \\
\hline $\mathrm{U}$ & -10.5 & -10.6 & -10.0 & -10.0 & -9.2 & -9.3 \\
\hline $\mathrm{Np}$ & - & - & -37.5 & -36.7 & -3.3 & -3.3 \\
\hline $\mathrm{Pu}$ & 10.7 & 11.5 & 13.2 & 13.6 & 15.7 & 16.5 \\
\hline $\mathrm{Am}$ & 263.5 & 263.2 & -35.6 & -34.8 & -4.7 & -4.6 \\
\hline $\mathrm{Cm}$ & - & - & 86.0 & 84.3 & 41.5 & 40.9 \\
\hline MA & 381.7 & 380.0 & -27.9 & -27.3 & -1.5 & -1.5 \\
\hline \multicolumn{7}{|c|}{ Mass balance $(\mathrm{kg} / \mathrm{TWhe})$ at EOL } \\
\hline $\mathrm{U}$ & -129.5 & -130.0 & -113.5 & -113.7 & -132.0 & -132.6 \\
\hline $\mathrm{Np}$ & 0.6 & 0.6 & -3.4 & -3.4 & -0.3 & -0.3 \\
\hline $\mathrm{Pu}$ & 17.9 & 19.2 & 22.0 & 22.8 & 26.2 & 27.5 \\
\hline $\mathrm{Am}$ & 3.5 & 3.5 & -14.8 & -14.5 & -1.9 & -1.9 \\
\hline $\mathrm{Cm}$ & 0.9 & 0.9 & 3.1 & 3.0 & 1.4 & 1.4 \\
\hline MA & 5.0 & 5.0 & -15.2 & -14.8 & -0.8 & -0.8 \\
\hline
\end{tabular}


- In the analysis with EVOLCODE, 11 axial levels were considered as a best estimate case ( 10 axial levels in the active core, $10 \mathrm{~cm}$ high each and one axial level at the bottom, for the lower blanket, $30 \mathrm{~cm}$ high). This proposal of meshing is a result of the CIEMAT team experience acquired in past exercises and it tries to capture the spatial influence on the spectra. Results are provided in Section 4. In addition, a case with two axial levels, exactly the same than SERPENT, will be provided in Section 5 to ease a direct code to code comparison. This case is also a sensitivity analysis for EVOLCODE.

The computational characteristics of the machines employed to run SERPENT and EVOLCODE are showed in Table 1.

\section{Core performances results with SERPENT and EVOLCODE}

Global transmutation performances as well as neutronics safety parameters for the three cases under study, CONF2 (reference with no MA transmutation strategy), HOM4 and HET2 are provided in this section. Here, the 11-axial-levels model in EVOLCODE is used.

\subsection{Evolution of Pu and $M A$}

Table 2 shows the core mass balances for the different cases. The transmutation rate (TR) presented in the table is defined as the relative mass produced from beginning of life (BOL, 0 EFPD) to end of life (EOL, $2050 \mathrm{EFPD})$, as:

$\mathrm{TR}(\%)=[\mathrm{M}(\mathrm{EOL})-\mathrm{M}(\mathrm{BOL})] / \mathrm{M}(\mathrm{BOL}) \cdot 100$.

Assuming a $40 \%$ thermodynamic efficiency, the minor actinide balance per electricity production is also provided.

It can be observed in Table 2 that all cases under study are breeders concerning the total plutonium mass balance (with $\mathrm{Pu}$ breeding ratio values of the order of 1.11 for CONF2, 1.15 for HOM4 and 1.17 for HET2, when averaged in the 820-1230 EFPD period). The total plutonium breeding is more important when MA are loaded, producing HET2 the highest amount of Pu due to the extra radial ring.

Fig. 3 shows the mass balance of Pu isotopes from BOL to EOL in the different core regions. ${ }^{239} \mathrm{Pu}$ is the main responsible of the increase of $\mathrm{Pu}$ mass in all configurations and accumulates in all regions, being its maximum production in the axial fertile blanket, as expected (it comes from captures in ${ }^{238} \mathrm{U}$ ). The ${ }^{238} \mathrm{Pu}$ mass increases in the cores loaded with MA, since it originates in chains starting in ${ }^{241} \mathrm{Am}$ and ${ }^{237} \mathrm{~Np}$.

Concerning the change of MA masses, there is a global accumulation in CONF2, a moderate destruction in HOM4 (with a net Am and $\mathrm{Np}$ destruction competing with $\mathrm{Cm}$ accumulation) and a slight but net MA destruction in HET2. The MA behaviour is also depicted in Fig. 4.

\section{2. $k_{\text {eff }}$ Comparison}

The $k_{\text {eff }}$ results along irradiation in the single batch approach are depicted in Fig. 5. It can be seen in the figure that addition of MA in a fresh core has a negative effect in reactivity at BOL, as the $k_{\text {eff }}$ is lower in HOM4 and HET2 configurations. Also, as a result of much more ${ }^{238} \mathrm{Pu}$ breeding capability in the HOM4 driver core, the positive reactivity swing is higher, of the order of $600 \mathrm{pcm}$ for the period 820-1230 EFPD.

\subsection{Reactivity coefficients}

Important information is required for safety analysis concerning reactivity coefficients, which have been calculated in the represen-
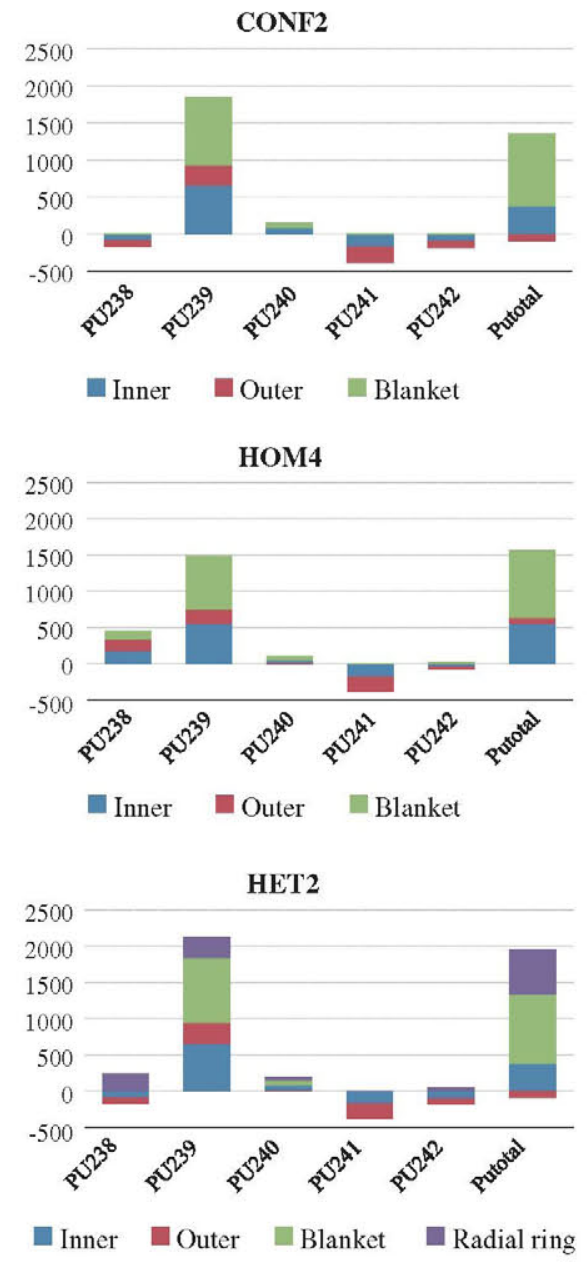

Fig. 3. Mass balance (kg) for Pu isotopes at EOL (EOL-BOL), SERPENT (very similar results were obtained with EVOLCODE).
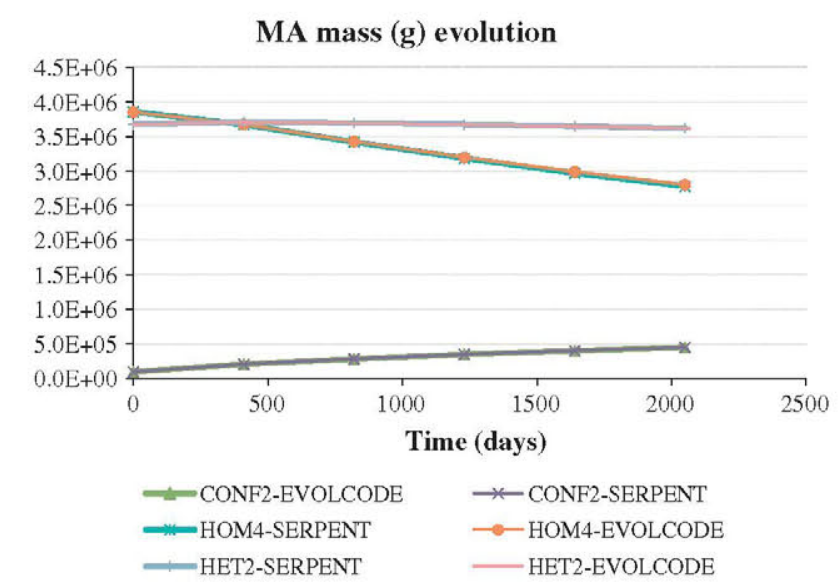

Fig. 4. Total minor actinides evolution.

tative equilibrium sub-cycle and are shown in Tables 3 and 4. The discrepancies between codes are less than $10 \%$.

The Doppler constant is the faster compensating safety parameter against positive reactivity insertions. Here, the Doppler constant estimation comes from the following equation:

$K_{\mathrm{d}}=\frac{\rho(2500)-\rho(1500)}{\ln 2500-\ln 1500}$ 


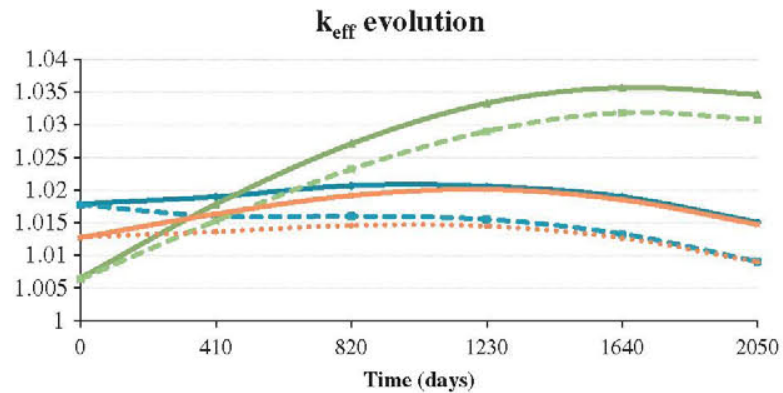

- CONF2-EVOLCODE $-\varpi-$ CONF2-SERPENT
- HOM4-EVOLCODE $-\cdots-$ HOM4-SERPENT
HET2-EVOLCODE $\cdots . .$. HET2-SERPENT

Fig. 5. $k_{\text {eff }}$ Evolution.

where $\rho(2500)$ is the reactivity when the fuel temperature has been increased by $1000 \mathrm{~K}$ and $\rho(1500)$ is the nominal reactivity value (the whole core fuel is assumed at $1500 \mathrm{~K}$ ). This approach was agreed among the CP-ESFR Project partners and is justified by the linear dependence of Doppler effect with logarithmic temperature increments. A more rigorous method for Doppler constant estimation should account for more temperature intervals below and above the $1500 \mathrm{~K}$ nominal one and data interpolation; however the procedure would require much time for comparison of codes.

In the HOM4 case the Doppler constant is lower than in CONF2 configuration due to the $4 \mathrm{wt} \%$ of $U$ content replaced by MA, being the broadening of ${ }^{238} \mathrm{U}$ capture cross section one of the main contributions to Doppler effect, while broadening of the substitute ${ }^{241} \mathrm{Am}$ takes place at much lower neutron energies, below the fast spectrum.

The effective delayed neutron fraction, $\beta_{\text {eff, }}$ has an important role in the power response to a transient. It has been calculated with the method developed by (Meulekamp and Van der Marck, 2006) as described in Section 3.1. $\beta_{\text {eff }}$ in the HOM4 configuration is only $6 \%$ worse than in the reference optimized configuration. In the HET2 case, its value is similar than in the reference case since the MA content is located only in a radial blanket around the active core. It has been estimated that $\beta_{\text {eff }}$ is reduced by 25 pcm during 2050 EFPD burnup.

Coolant density effect is a challenging parameter in large fast reactor design due to its large positive value. Coolant voiding in the core has three effects in reactivity: a positive contribution because of spectral hardening (increase of the ${ }^{239} \mathrm{Pu}$ fission to ${ }^{238} \mathrm{U}$ capture rate at higher energies); a negative contribution as a result of neutron leakage increase depending on the reactor size and on the height to radius rate; and finally a positive contribution as a result of decrease in coolant captures. In the case of commercial reactors like the ESFR concept the void worth has a global positive value. In the optimized configuration presented in this paper the sodium plenum above the core increase leakages if a bubble occurs in this zone, while the upper absorber layer avoids backscattering to the active region.

The void worth is the reactivity difference between the voided and the reference core. Core void worth represents the worst possible scenario and has been calculated by decreasing Na density to $10^{-10} \mathrm{~g} / \mathrm{cm}^{3}$ between rods in the $1 \mathrm{~m}$ active length (wrapper interstitials, control rod channels and steel reflectors were not voided). It may be seen in the tables that discrepancies between codes are less than $2 \%$. The core void worth has a value around $4.5 \$$ for CONF2 and HET2 configurations and around $5 \$$ for the HOM4 option. Core void worth slightly increases from BOEC to EOEC.

In addition, an extended reactor void worth has been calculated, where not only the coolant in the core is voided, but also the upper part until the sodium plenum, that is, active length, upper fission gas plenum, upper plug and upper sodium plenum. In the HET2 configuration the radial blanket is also emptied. The extended void worth has a value around $2.5 \$$, clearly more easy to compensate with the negative reactivity coefficients such as the Doppler and thermomechanical expansion of the core.

\subsection{Linear power}

Complementary information obtained with EVOLCODE is provided in this section concerning the linear power, a major core design parameter. The middle plane distribution as a function of burnup is shown in Fig. 6 for the CONF2 case. At beginning of life the higher power peak is in the outer core due to the higher Pu content in this zone. Due to the higher flux in the outer core the $\mathrm{Pu}$ is burned faster and at EOL the power has a more flattened shape. Fig. 7 is a comparison of all three cases at BOL conditions.

\section{Detailed comparison of EVOLCODE and SERPENT}

A detailed comparison between EVOLCODE and SERPENT codes is now explained in this section. The benchmark is carried out for the HOM4 case using the same axial nodalization in both codes:

Table 3

Reactivity coefficients at pseudo-BOC (820 EFPD).

\begin{tabular}{|c|c|c|c|c|c|c|}
\hline & \multicolumn{2}{|l|}{ CONF2 } & \multicolumn{2}{|l|}{ HOM4 } & \multicolumn{2}{|l|}{ HET2 } \\
\hline & SERPENT & EVOLCODE & SERPENT & EVOLCODE & SERPENT & EVOLCODE \\
\hline Doppler, pcm & -891 & -827 & -562 & -594 & -762 & -783 \\
\hline$\beta_{\text {eff }}$ & 373 & 370 & 350 & 346 & 372 & 368 \\
\hline Core void worth, pcm & $1476(3.96 \$)$ & $1516(4.10 \$)$ & $1714(4.9 \$)$ & $1712(4.95 \$)$ & $1527(4.11 \$)$ & $1517(4.12 \$)$ \\
\hline Extended void worth, pcm & $719(1.93 \$)$ & $767(2.07 \$)$ & $1036(2.96 \$)$ & $1061(3.07 \$)$ & $781(2.1 \$)$ & $743(2.02 \$)$ \\
\hline
\end{tabular}

Table 4

Reactivity coefficients at pseudo-EOC (1230 EFPD).

\begin{tabular}{|c|c|c|c|c|c|c|}
\hline & \multicolumn{2}{|l|}{ CONF2 } & \multicolumn{2}{|l|}{ HOM4 } & \multicolumn{2}{|l|}{ HET2 } \\
\hline & SERPENT & EVOLCODE & SERPENT & EVOLCODE & SERPENT & EVOLCODE \\
\hline Doppler, pcm & -727 & -772 & -570 & -629 & -723 & -717 \\
\hline$\beta_{\mathrm{eff}}$ & 367 & 362 & 345 & 338 & 365 & 359 \\
\hline Core void worth, pcm & $1636(4.5 \$)$ & $1654(4.6 \$)$ & $1778(5.2 \$)$ & $1746(5.2 \$)$ & $1622(4.5 \$)$ & $1626(4.5 \$)$ \\
\hline Extended void worth, pcm & $896(2.4 \$)$ & $922(2.5 \$)$ & $1145(3.3 \$)$ & $1095(3.2 \$)$ & $907(2.5 \$)$ & $875(2.4 \$)$ \\
\hline
\end{tabular}




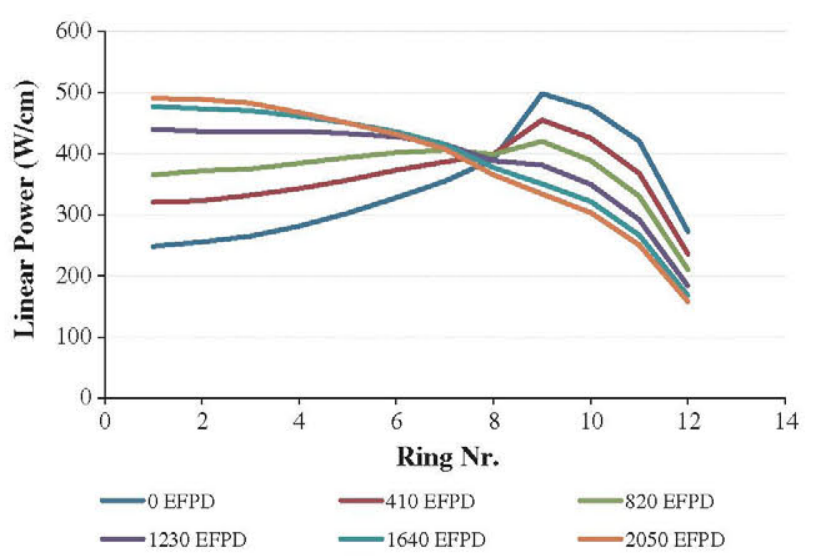

Fig. 6. Linear power radial profile evolution with the burnup, central core plane, CONF2 case.

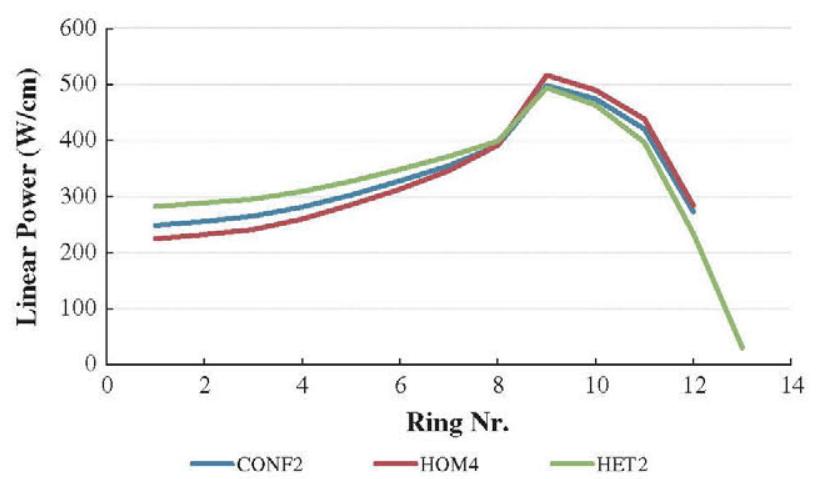

Fig. 7. Comparison of linear power radial profile in the central core plane, at BOL.

one for the active core and one for the axial blanket, while the same 12 radial rings are preserved.
Isotope mass differences are provided in Table 5, second to seventh column. It can be seen that the agreement is very good, being the differences lower than $3 \mathrm{wt} \%$ in all cases. An important contribution to discrepancies takes place already in the first irradiation step, 0-410 EFPD.

As explained in Section 3 a different treatment of the branching ratios was observed in SERPENT and EVOLCODE. In fact, the last column in Table 5 shows results when the SERPENT default branching ratio is used. In this case, EOL differences concerning some $\mathrm{Am}$ and $\mathrm{Cm}$ isotopes (as well as ${ }^{238} \mathrm{Pu}$ ) were much higher. As a consequence of the reaction capture in ${ }^{241} \mathrm{Am}$, isotopes ${ }^{242} \mathrm{Am}$ and ${ }^{242 \mathrm{~m}} \mathrm{Am}$ appear with a fraction given by the reaction branching ratio. ${ }^{242} \mathrm{Am}$ then decays with a short half-life into ${ }^{242} \mathrm{Cm}(16 \mathrm{~h})$ and ${ }^{242 \mathrm{~m}} \mathrm{Am}$ is a fissile isotope with a long life (141 years). When the SERPENT constant value was modified to employ a spectrum-averaged value, similar to the EVOLCODE one (fraction to the isomeric state equal to 0.133 ), Am and $\mathrm{Cm}$ isotopes exhibit comparable differences in both codes as shown second to seventh column of Table 5 .

Fig. 8 shows the $k_{\text {eff }}$ during the total irradiation period. The difference between codes increases in each burn step with a maximum value of $630 \mathrm{pcm}$ at $2050 \mathrm{EFPD}$. It is interesting to observe that EVOLCODE always predicts a higher reactivity than SERPENT, as the Pu content estimation at the end of each step is higher with EVOLCODE. Fig. 9 shows total mass differences between codes in ${ }^{239} \mathrm{Pu}$ and ${ }^{238} \mathrm{U}$ isotopes. In addition, in Fig. $8 k_{\text {eff }}$ results for the 10 axial levels case are also plotted. In this case the sensitivity is some $300 \mathrm{pcm}$ at EOL between both EVOLCODE cases, although the agreement is very good until 1230 EFPD.

Looking for potential explanations of discrepancies between SERPENT and EVOLCODE, the burnup methodology arises as the main candidate. Firstly, it has been checked that concerning the normalization issue, both codes differ very little in their fission Q-value assumptions. On the other hand, although both codes use approaches that belong to the group of predictor-corrector methods, the algorithms employed are not the exactly the same (as explained in Sections 3.1 and 3.2). The effect is illustrated in

Table 5

Percentage relative errors [(SERPENT-EVOLCODE)/EVOLCODE] in the prediction of isotopes masses along irradiation, HOM4 case.

\begin{tabular}{|c|c|c|c|c|c|c|c|}
\hline EFPD & $\begin{array}{l}0 \\
(\mathrm{BOL})\end{array}$ & 410 & $\begin{array}{l}820 \\
(\mathrm{BOC})\end{array}$ & $\begin{array}{l}1230 \\
(\mathrm{EOC})\end{array}$ & 1640 & $\begin{array}{l}2050 \\
(\mathrm{EOL})\end{array}$ & $\begin{array}{l}2050 \\
\text { (Default branching ratio) }\end{array}$ \\
\hline U235 & 0.01 & -0.27 & -0.60 & -0.90 & -1.12 & -1.29 & -1.35 \\
\hline U236 & & 1.34 & 1.32 & 1.16 & 0.91 & 0.66 & \\
\hline U237 & & 0.96 & 1.60 & 1.90 & 1.78 & 1.77 & \\
\hline U238 & 0.02 & 0.03 & 0.03 & 0.04 & 0.05 & 0.05 & 0.04 \\
\hline Np237 & 0.03 & -0.16 & -0.37 & -0.56 & -0.71 & -0.83 & -0.88 \\
\hline $\mathrm{Np} 238$ & & 1.59 & 1.67 & 1.29 & 0.73 & 0.52 & 0.60 \\
\hline Np239 & & -0.78 & -0.52 & -0.42 & -0.45 & -0.48 & -0.39 \\
\hline Pu238 & 0.02 & 0.52 & 0.92 & 1.05 & 1.00 & 0.86 & 2.02 \\
\hline Pu239 & 0.02 & -0.26 & -0.44 & -0.60 & -0.77 & -0.92 & -0.91 \\
\hline Pu240 & 0.02 & 0.01 & -0.01 & -0.02 & -0.03 & -0.06 & -0.06 \\
\hline Pu241 & 0.03 & -0.04 & -0.10 & -0.12 & -0.09 & -0.07 & -0.09 \\
\hline Pu242 & 0.03 & -0.05 & -0.12 & -0.20 & -0.30 & -0.39 & -0.16 \\
\hline Pu243 & & 0.69 & 0.35 & 0.11 & -0.09 & -0.35 & 0.83 \\
\hline Am241 & 0.02 & -0.19 & -0.43 & -0.64 & -0.80 & -0.91 & -0.97 \\
\hline Am242 & & 1.66 & 1.56 & 1.31 & 0.51 & 0.26 & 2.39 \\
\hline $\operatorname{Am} 242 \mathrm{M}$ & 0.34 & 1.20 & 1.15 & 0.84 & 0.39 & -0.01 & -12.96 \\
\hline $\operatorname{Am} 243$ & 0.02 & -0.10 & -0.21 & -0.32 & -0.42 & -0.50 & -0.65 \\
\hline $\mathrm{Cm} 242$ & -0.90 & 2.19 & 2.19 & 1.84 & 1.27 & 0.86 & 3.03 \\
\hline $\mathrm{Cm} 243$ & 1.18 & 1.62 & 2.23 & 2.55 & 2.53 & 2.14 & 4.13 \\
\hline $\mathrm{Cm} 244$ & 0.04 & 0.34 & 0.49 & 0.54 & 0.51 & 0.44 & 0.42 \\
\hline $\mathrm{Cm} 245$ & 0.06 & -0.05 & -0.04 & 0.03 & 0.10 & 0.13 & 0.14 \\
\hline $\mathrm{Cm} 246$ & 0.72 & 0.85 & 0.94 & 1.02 & 1.08 & 1.17 & 1.20 \\
\hline $\mathrm{U}$ & 0.02 & 0.04 & 0.04 & 0.04 & 0.04 & 0.05 & 0.05 \\
\hline $\mathrm{Np}$ & 0.03 & -0.17 & -0.38 & -0.56 & -0.70 & -0.82 & -0.86 \\
\hline $\mathrm{Pu}$ & 0.02 & -0.11 & -0.21 & -0.28 & -0.38 & -0.48 & -0.39 \\
\hline Am & 0.03 & -0.15 & -0.34 & -0.52 & -0.66 & -0.77 & -1.36 \\
\hline $\mathrm{Cm}$ & 0.06 & 0.72 & 0.81 & 0.75 & 0.62 & 0.50 & 0.81 \\
\hline
\end{tabular}




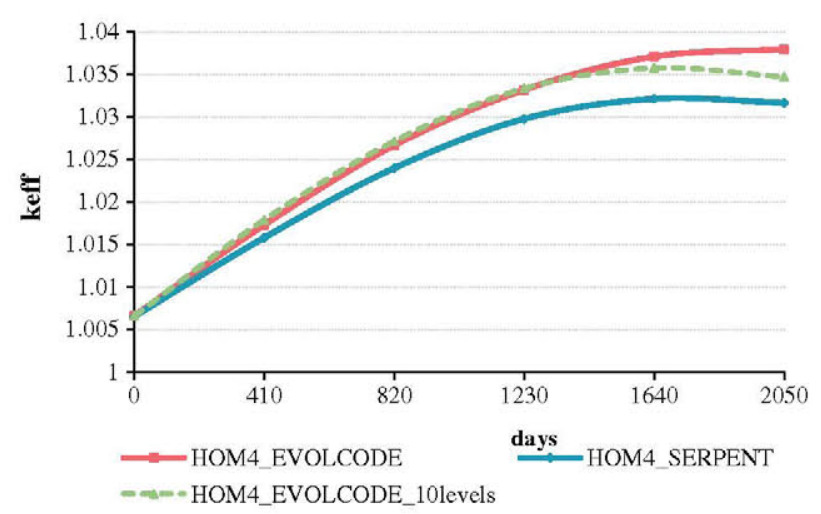

Fig. 8. $k_{\text {eff }}$ Comparison in HOM4 case, SERPENT and EVOLCODE (EVOLCODE results for 10 axial levels for the active core is also included).

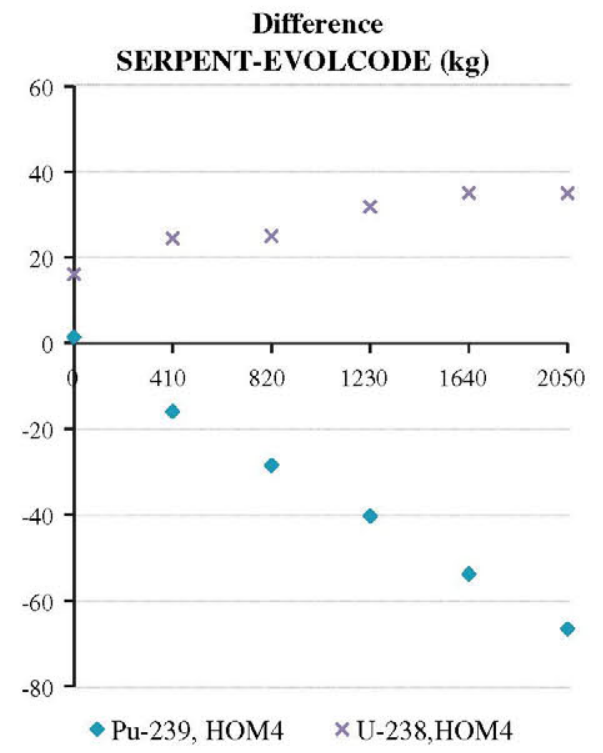

Fig. 9. Total mass difference between SERPENT and EVOLCODE for ${ }^{239} \mathrm{Pu}$ and ${ }^{238} \mathrm{U}$ (HOM4).

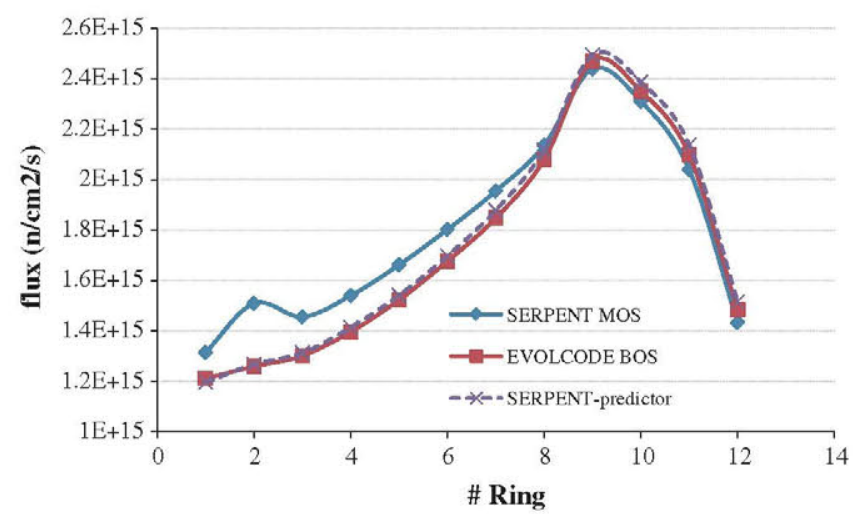

Fig. 10. Flux used for depletion in EVOLCODE and SERPENT in the first burnup step $(0-410$ EFPD).

Fig. 10 which shows total flux values employed for both codes in the first burnup step, where core composition significantly changes starting from fresh fuel. While SERPENT averages out the fluxes from the predictor and corrector method (SERPENT MOS in the figure) to obtain the definite value, the EVOLCODE definitive value
Table 6

Percentage relative errors [(EVOLCODE (fine time step)-EVOLCODE (coarse time step))/EVOLCODE (coarse time step)] in the prediction of isotopes masses along irradiation, HOM4 case.

\begin{tabular}{llrrrrr}
\hline EFPD & 0 & \multicolumn{1}{l}{410} & \multicolumn{1}{l}{$\begin{array}{l}820 \\
\text { (BOC) }\end{array}$} & $\begin{array}{r}1230 \\
\text { (EOC) }\end{array}$ & \multicolumn{1}{l}{1640} & $\begin{array}{r}2050 \\
(\mathrm{EOL})\end{array}$ \\
\hline U235 & 0.00 & -0.06 & -0.11 & -0.14 & -0.10 & -0.03 \\
U236 & & 0.23 & 0.21 & 0.15 & 0.02 & -0.09 \\
U237 & & 0.15 & 0.18 & -0.21 & 0.14 & -0.21 \\
U238 & 0.00 & -0.01 & -0.02 & -0.03 & -0.03 & -0.03 \\
Np237 & 0.00 & -0.02 & -0.04 & -0.05 & -0.01 & 0.03 \\
Np238 & & -0.25 & -0.30 & -0.32 & -0.55 & -0.34 \\
Np239 & & 0.10 & 0.09 & 0.16 & 0.08 & 0.17 \\
Pu238 & 0.00 & 0.08 & 0.13 & 0.13 & 0.05 & -0.04 \\
Pu239 & 0.00 & 0.15 & 0.25 & 0.32 & 0.34 & 0.34 \\
Pu240 & 0.00 & 0.00 & 0.01 & 0.03 & 0.06 & 0.08 \\
Pu241 & 0.00 & 0.00 & 0.01 & 0.04 & 0.07 & 0.11 \\
Pu242 & 0.00 & 0.01 & 0.03 & 0.04 & 0.04 & 0.05 \\
Pu243 & & -1.00 & -1.04 & -1.07 & -0.93 & -0.74 \\
Am241 & 0.00 & -0.04 & -0.07 & -0.07 & -0.02 & 0.05 \\
Am242 & & -0.12 & -0.23 & -0.35 & -0.66 & -0.46 \\
Am242M & 0.00 & 0.19 & 0.14 & 0.02 & -0.16 & -0.29 \\
Am243 & 0.00 & -0.04 & -0.07 & -0.10 & -0.12 & -0.13 \\
Cm242 & 0.00 & 0.32 & 0.23 & 0.08 & -0.26 & -0.39 \\
Cm243 & 0.00 & -0.14 & 0.05 & 0.23 & 0.11 & -0.19 \\
Cm244 & 0.00 & 0.04 & 0.03 & 0.00 & -0.06 & -0.13 \\
Cm245 & 0.00 & -0.02 & -0.02 & 0.00 & 0.00 & -0.03 \\
Cm246 & 0.00 & 0.08 & 0.10 & 0.13 & 0.14 & 0.16 \\
\hline
\end{tabular}

(EVOLCODE BOC in the figure) is very similar to the SERPENT predictor value, i.e., previous to correction. As a consequence, differences in the definitive flux estimations will lead to differences in compositions.

Finally, the burnup time step size has been also investigated with EVOLCODE to demonstrate that the influence on isotope masses and $k_{\text {eff }}$ is very small. An additional case was calculated with a smaller size, 51.25 EFPD (fine time step) to compare results with the original 410 EFPD (coarse time step) case. The found differences in terms of actinide masses are shown in Table 6 . When compared to coarse time step results, differences are lower than $0.8 \%$ at EOL (the higher value is for Pu-243, having a short half-life of $4.96 \mathrm{~h}$ ). Concerning $k_{\text {eff }}$, differences were $100 \mathrm{pcm}$ at EOL. Therefore, it might be concluded that the use of a finer time mesh has a minimal impact on the isotopics and $k$-effective.

\section{Conclusions}

A detailed analysis of the optimized CP-ESFR CONF2 core concept has been done with two last generation fuel depletion Monte Carlo codes for two different MA loading strategies. Conclusions are provided under two points of view: (i) core performances and (ii) computer codes comparison.

(i) All cases under analysis lead to total plutonium production as a result of the lower axial blanket (CONF2), the axial and radial blankets (HET2) or the lower axial blanket and MA loading in the driver region (HOM4). Total plutonium breeding ratios range from 1.1 to 1.17 .

Concerning MA behaviour, the reference CONF2 core is a net MA producer (some 4.5 times more than the small initial and unintended loading).

The HOM4 core configuration leads to the net elimination of an important amount of MA, specifically americium and neptunium. Concerning curium, there is a net accumulation in spite of the important amount of $\mathrm{Cm}$ fissioning. As the management of curium entails difficulties and costs, a careful analysis of possibilities taking into account ${ }^{244} \mathrm{Cm}$ (18.11 years half-life) 
during storage is required. Compared to a similar core with no MA loading, the HOM4 core void deteriorates some $12 \%$ and the extended void some $20 \%$.

On the other hand, HET2 model presents similar safety characteristics than the CONF2 and a nearly neutral behaviour regarding MA transmutation (in fact, a small destruction is estimated). Even though detailed transient analysis is still needed in order to conclude the licensing of such cores, the estimated safety coefficients are encouraging. Even for HOM4 core, the core void is some $5.2 \$$, while extended void is some $3.3 \$$.

(ii) In what concerns comparison of codes, a great special care was taken to develop BOL similar models: same core geometries, initial fuel and material compositions and masses, neutron libraries and working temperatures. Then, results predicted by EVOLCODE and SERPENT codes are very close in $k_{\text {eff }}$ estimations before activation of burnup models. After a typical long irradiation period of 2050 EFPD, when a similar reasonable assumption is taken concerning the branching ratio, discrepancies are quantified in the order of $3 \%$ concerning actinide isotope masses, $600 \mathrm{pcm}$ as much in $k_{\text {eff }}$, and $10 \%$ concerning reactivity parameters. The different corrector-predictor approaches in the fuel depletion model most likely explain the observed differences.

\section{Acknowledgements}

This work is partially funded by the EC Commission under the 7th EURATOM Framework Program, within the Collaborative Project CP-ESFR "European Sodium Fast Reactor". In addition, authors would like to acknowledge the Spanish Consejo de Seguridad Nuclear for having partially funded this work and ENRESA also for its partial support in the frame of CIEMAT-ENRESA agreement.

\section{References}

Âlvarez-Velarde, F., León, P.T., González-Romero, E.M., 2007. EVOLCODE2, a combined neutronics and burn-up evolution simulation code. In: Proc. 9th Information Exchange Meeting on Actinide and Fission Product P\&T (Nîmes, France). ISBN 978-92-64-99030-2, NEA/OECD.

Âlvarez-Velarde, F., 2011. Development of a Computational Tool for the Simulation of Innovative Transmutation Systems. PhD thesis, University of Córdoba, Spain.

Âlvarez-Velarde, F., González-Romero, E.M., 2012. Sensitivity/uncertainty analysis of the validation of the EVOLCODE 2.0 burn-up system with PWR experimental data. In: 12th IEM on Actinide and Fission Product Partitioning and Transmutation (12-IEMPT), NEA/OECD, Prague, Czech Republic, 24-27 September 2012.

Bays, S.E., Herring, J.S., Tulenko, J., 2011. An axially heterogeneous sodium-cooled fast reactor designed to transmute minor actinides. Nuclear Technology 173 , 115-134.

Buiron, L., Varaine, F., Lorenzo, D., Palancher, H., Valentin, B., 2007. Minor actinides transmutation in SFR depleted uranium radial blanket, neutronic and thermal hydraulic evaluation. In: Proceedings of Global 2007, Boise, Idaho, September 9-13.

Buiron, L., Varaine, F., Verrier, D., Ruah, D., Massara, S., Garzenne, C., 2009 Heterogeneous minor actinide transmutation on a UO2 blanket and on (U,Pu)O2 fuel in a sodium-cooled fast reactor - assessment of core performances. In: Proceedings of Global 2009, paper 9109, Paris, September 6-11.

Fensin, M.L., Hendricks, J.S., Anghaie, S., 2010. The enhancements and testing for the MCNPX 2.6.0 depletion capability. Nuclear Technology 170, 68-79.

Fiorini, G.L., Vasile, A., 2011. European commission - 7th framework programme. the collaborative project on European sodium fast reactor (CP ESFR). Nuclear Engineering and Design 241, 3461-3469.

Isotalo, A., Aarnio, P.A., 2011a. Comparison of depletion algorithms. Ann. Nucl. Energy. 38 (2-3), 261-268.

Isotalo, A., Aarnio, P.A., 2011b. Higher order methods for burnup calculations with Bateman solutions. Ann. Nucl. Energy 38 (1987-1995).

Leppänen, J., 2011. PSG2/Serpent - A Continuous-energy Monte Carlo Reactor Physics Burnup Calculation Code, User's Manual. VTT Technical Research Center of Finland.

Leppänen, J., Isotalo, A., 2012. Burnup calculation methodology in the serpent 2 Monte Carlo Code. In: Proc. PHYSOR-2012. Knoxville, TN, 15-20 April, 2012.

Meulekamp, R.K., van der Marck, S.C., 2006. Calculating the effective delayed neutron fraction with Monte Carlo. Nucl. Sci. Eng. 152 (2006), 142-148.

Moore, R.L., et al., 1995. MOCUP:MCNP-ORIGEN2 Coupled Utility Program INEL-95/ 0523, Idaho National Engineering Laboratory.

Naganuma, M., Ogawa, T., Ohki, S., Mizuno, T., Kotake, S., 2010. Minor actinidebearing oxide fuel core design study for the JSFR. Nuclear Technology 170, 170180.

OECD/NEA, 2004. Pyrochemical Separations in Nuclear Applications. A Status Report. NEA Report 5427.

OECD/NEA, 2010. National Programmes in Chemical Partitioning. A Status Report. NEA Report 5425.

OECD/NEA, 2012. Curium Management Studies in France, Japan and USA. NEA/NSC/ WPFC/DOC(2012)2 (January).

ohki, S., Sato, I., Mizuno, T., Hayashi, H., Tanaka, K., 2007. An effective loading method of americium targets in fast reactors. In: Proceedings of Global 2007 Boise, Idaho, USA, September 9-13, 2007.

Palmiotti, G., Salvatores, M., Assawaroongruengchot, M., 2011. Impact of the core minor actinide content on fast reactor reactivity coefficients. Journal of Nuclear Science and Technology 48 (4), 628-634.

Pelowitz, D.B., ed. 2008. MCNPX User's Manual, Version 2.6.0. LA-CP-07-1473 (April).

Pusa, M., Leppänen, J., 2010. Computing the matrix exponential in burnup calculations. Nucl. Sci. Eng. 164 (2), 140-150.

Pusa, M., 2011. Rational approximations to the matrix exponential in burnup calculations. Nucl. Sci. Eng. 169 (2), 155-167.

Rimpault, et al., 2002. The ERANOS code and data system for fast reactor neutronics analyses. In: Proc. PHYSOR 2002 Conf., Seoul, Korea, October 2002.

Rineiski, A., et al. 2011. Synthesis of options to optimize feedback coefficients, SP2.1.3.D1. CP-ESFR Project.

RSICC Computer Code Collection-ORIGEN 2.1, 1999. Isotope Generation and Depletion Code Matrix Exponential Method, CCC-371. Oak Ridge National Laboratory.

Sanz, J., Cabellos, O., García-Herranz, N., 2008. ACAB Inventory Code for Nuclear Applications: User's Manual V.

Trellue, H.R., Poston, D.I., 1999. User's Manual, Version 2.0 for MONTEBURNS Version 5B, LA-UR-99-49999, Los Alamos National Laboratory.

$\mathrm{Xu}, \mathrm{Z}$., Hejzlar, P., 2008. MCODE, Version 2.2: An MCNP-ORIGEN Depletion Program. MIT-NFC-TR-104, Massachusetts Institute of Technology.

Yang, W.S., 2012. Fast reactor physics and computational methods. Nuclear Engineering and Technology 44 (2).

Zhang, Y., Wallenius, J., Fokau, A., 2010. Transmutation of americium in a medium size sodium cooled fast reactor design. Annals of Nuclear Energy 37, 629-638. 